\title{
Messen, was nicht messbar ist
}

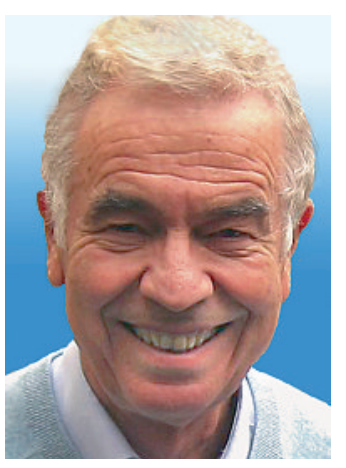

Hans Stalder
Ständig messen, zählen und berechnen wir. Wir messen Grösse und Gewicht und errechnen damit den BMI. Wir messen Blutdruck, Cholesterinwerte und Knochendichte, um die Wahrscheinlichkeit einer zukünftigen Krankheit zu berechnen. Und unseren Diagnosen liegen schliesslich Berechnungen von Werten zugrunde, die Anamnese, Status und die weiteren Untersuchungen ergeben haben.

Selbstverständlich schätzen die Patienten kompetente Ärzte, die eine messbare und quantifizierbare Medizin betreiben. Allerdings belegen praktisch alle Studien zur Patientenzufriedenheit [1], dass Patienten vor allem die «Menschlichkeit» des Arztes schätzen Empathie und einfühlsame Kommunikation. Genügend Zeit für die Konsultation, präventive Massnahmen, unkomplizierter Zugang zur Praxis und Kontinuität der Behandlung sind weitere wichtige Desiderata.

\section{Praktisch alle Studien zur Patientenzufriedenheit belegen, dass die Patienten vor allem die Menschlich- keit des Arztes schätzen - Empathie und einfühlsame} Kommunikation

\footnotetext{
* Prof. Dr. med. Hans Stalder, Facharzt FMH für Innere Medizin FMH, Redaktionsmitglied, ist ehemaliger Chefarzt der Policlinique de Médecine et du Département de Médecine communautaire des Hôpitaux Universitaires de Genève.
}

1 Wensing M, Jung HP, Mainz J, Olesen F, Grol R. A systematic review of the literature on patient priorities for general practice care. Part 1: Description of the research domain. Soc Sci Med. 1998; 47:1573-88.
Aus meiner Sicht ist die Unzufriedenheit der Hausärzte zum Teil darauf zurückzuführen, dass sie für ganz zentrale Leistungen, die sie in ihrem Beruf erbringen, keine Vergütung erhalten und somit keine Wertschätzung erfahren. Mit TARMED kann nur fakturiert werden, was messbar ist: technische Leistungen und Zeit. Diese Abrechnungsmethode ist für fachärztliche Leistungen durchaus sinnvoll. So ist zum Beispiel die Kostenkalkulation für eine Koloskopie relativ einfach: Man misst die Dauer der Intervention, die Abschreibung des Gerätes und legt den Preis fest. Hausärzte, die wenig technische Leistungen durchführen, werden vor allem lich nicht falsch, denn auch die Patienten schätzen die Zeit, die man ihnen widmet. Allerdings ist die Gesprächsdauer allein weder ein Mass für die Empathie noch für die Qualität der Konsultation.

Und wie sieht es im Spital aus? Es ist zu bezweifeln, dass die Fakturierung nach Diagnosen (DRG) dem besser gerecht wird, was wirklich wichtig ist und den Wünschen der Patienten am meisten entspricht: eine hohe Behandlungsqualität. Denn dazu gehören nicht nur die Überlebensraten nach einer Operation auf Basis eines bestimmten Diagnosecodes oder die nach Konsultationsdauer bezahlt. Das ist grundsätz-
Rehospitalisierungsraten - die alle kalkulierbar sind sondern ebenso und insbesondere die Qualität der Betreuung, die Kommunikation und die Empathie des Pflegepersonals. Auch hier ist die (späte) Frustration einiger Ärzte und Pflegefachleute nachvollziehbar, denn die neue Abrechnungsmethode nach DRG vernachlässigt soziale und menschliche Faktoren und berücksichtigt eben nicht, was nicht messbar ist. Man kann sich im Nachhinein fragen, ob die Berechnung nach Aufenthaltsdauer trotz aller Mängel die Behandlungsqualität nicht besser berücksichtigt hat als die Diagnosenpauschalen.

In Rechnung gestellt wird also nur das Quantifizierbare. Das ist aber nicht, was sich die Patienten am meisten wünschen. Die Manie, alles quantifizieren zu wollen, um es in Taxpunkte umwandeln zu können, birgt überdies noch andere Risiken. Bleibt das nicht Mess- und Zählbare unberücksichtigt, sind die Ärzte gezwungen, sich auf die fakturierbaren Leistungen zu konzentrieren wie Labortarife oder die Selbstdispensation. Ihre Grundwerte bleiben dabei auf der Strecke. Alles quantifizieren $\mathrm{zu}$ wollen, beschert uns darüber hinaus einen zunehmend aufgeblähten Verwaltungsapparat. Denn was man zählt, muss kontrolliert, und was man kontrolliert, muss überwacht werden.

Wie könnte man das ändern? Die Allgemeinmediziner sollten für eine angemessene Vergütung ihrer Schlüsselleistungen kämpfen, d.h. einer ganzheitlichen, patientenorientierten Behandlung. Leider scheint es keine Patentlösung zu geben. Auf den ersten Blick wird eine Fakturierung pro Kopf dieser Forderung besser gerecht, denn sie würde statt Leistung oder Diagnose die gesamte Person berücksichtigen. Die Pro-Kopf-Vergütung ist in anderen Ländern eingeführt worden, auch in der Schweiz im Rahmen von Managed-Care-Modellen. Aber nicht alle Ärzte sind mit der damit einhergehenden, notwendigerweise strikten Qualitätskontrolle einverstanden.

Und in den Spitälern? Wurde je über eine Fakturierung pro Kopf bzw. nach Anzahl der versorgten Einwohner nachgedacht? Selbstverständlich würde auch dies eine strikte Qualitätskontrolle voraussetzen. Gleichzeitig würde der riesige Verwaltungsapparat verkleinert mit unzähligen, Millionen verschlingenden Diagnose-Kodierern, die ihrerseits wieder kontrolliert und überwacht werden müssen. Und wir könnten uns endlich wieder auf das konzentrieren, was wirklich wichtig ist ...

Hans Stalder* 